\title{
Probing defects in friction stir welding process using temperature profile
}

\author{
B DAS, S PAL and S BAG*(1) \\ Department of Mechanical Engineering, Indian Institute of Technology Guwahati, Guwahati 781039, India \\ e-mail: swarupbag@iitg.ac.in
}

MS received 2 April 2016; accepted 9 January 2019; published online 14 March 2019

\begin{abstract}
Detection of defects in friction stir welding process is a challenging task since most of the defects are internal or subsurface. An attempt has been made to explore the methodologies based on temperature signal for possible detection of defects in friction stir welding process using different tool profiles. The presence of defect is affected by temperature diffusion and is better reflected by the change of temperature over time. Temperature signals are acquired using thermocouples inserted in specific locations on advancing and retreating side of the welds. The rate of change of temperature and wavelet-analysis-based indicator computed from temperature signal against each experiment reveal appreciable difference for defective and defect-free welds. Threshold values are computed that clearly set a boundary for classifying the defective welds from defect-free welds. The proposed approaches can effectively reduce the post-processing steps essential for other nondestructive evaluation of the welds and can work as a first level of safeguard in the identification of defects during friction stir welding process. The methodologies can be extended towards monitoring of the process in industrial applications.
\end{abstract}

Keywords. Defect detection; temperature profile; temperature gradient; wavelet analysis; monitoring.

\section{Introduction}

Deviation from the suitable welding window for a particular material can lead to the formation of defects in friction stir welding (FSW) process [1]. Defects in FSW process are mostly subsurface and need special arrangements for their detection. Destructive testing methods are useful in detecting internal or subsurface defects, but they are not suitable in the industrial environment. The difficulty of destroying the welded component can be overcome by implementing available non-destructive methods. These methods are efficient and can reveal most accurate results for identification of defects. Although the non-destructive methods are efficient they demand high initial investment and human intervention for interpretation of the results, and the analysis depends on the expertise of the operator.

Detection of defects in FSW process was attempted by researchers through Fourier analysis and artificial intelligence of real-time force data [2]. Frequency analysis of the force signals yields notable information for the classification of defective welds from defect-free welds. Successful application of X-ray radiography and infrared thermography techniques for detection of defects during FSW process of aluminium alloy in lap joint configuration [3] was

*For correspondence reported. One of the remarkable observations was that the decay of temperature is lower in case of defective joints compared with defect-free joints. Typical welding defects found in FSW process were analysed using optical microscopy, energy-dispersive X-ray spectroscopy and scanning electron microscopy [4]. Effect of tool tilt angle was investigated in the formation of defects. A new eddy current probe for the evaluation of defects in friction-stirwelded samples was designed by Rosado et al [5]. Based on the electrical signal acquired with the help of the developed probe, internal defects in the welds can be characterized with better accuracy. Implementation of other non-destructive methods for defect identification in FSW process was also reported in the technical articles [6-9]. These nondestructive methods yield satisfactory results in detection of subsurface defects, but limitations that prevail are high cost and precise expertise for the interpretation of data obtained. Hence, despite being accurate, these methods are still not accessible to many industrial applications.

Monitoring of other welding processes with real-time data has already gained significant attention [10-13]. Realtime data in monitoring of FSW process gain significant attention as physics involved in the process limits accurate phenomenological modelling of the process. Force signals [14-16], acoustic emission signals [17, 18] and driving motor current signal $[19,20]$ are a few real-time data that attracted researchers for fruitful implementation in the 
monitoring of FSW process. Apart from the real-time signal information, recently image-based analysis is also making significant progress in the detection of defects and monitoring of FSW process. The researchers considered top surface image of friction-stir-welded samples and analysed using discrete wavelet (DW) transform for classifying the defective and defect-free welds [21]. The extended fractal theory as a tool for analysing real-time speed signals acquired during FSW process is used for possible defect identification in the welded samples [22]. Computed fractal dimensions show appreciable deviation for defective and defect-free cases. The use of temperature signal for identification of defect formation in FSW process was reported [23]. Temperature data were computed through numerical simulation and an observer-based model was developed for detection of defects. The approach presented is appreciable but the accuracy of the developed model was not reported. Moreover, assumptions made during computation of temperature signal are not very clear when it compares with real-time welding operation.

Literature indicates that thermal properties are strongly influenced by the presence of micro- or macro-scale defects within the materials [4]. The defects mainly impact on effective thermal conductivity and in effect, the thermal diffusivity of the material [24]. However, microscopically defective materials are considered as homogenous structure in continuum scale. Therefore, presence of any material discontinuity (defect) influences the rate of transfer of heat, which is physically represented by the property of thermal diffusivity, assuming that this thermal property is a measurable quantity in macroscopic scale, which is represented by response temperature. Any variation in macroscopic properties is reflected by the measured temperature. However, the rate of change of temperature is more sensitive to represent the rate of heat transfer than only temperature. It is obvious that the variability in response of temperature diminishes away from the position of the defect [5]. Thus, analysis of measured temperature signal can signify the presence of any possible defects.

The aforementioned literature survey fetched the information that monitoring as well as defect identification is attempted by researchers in real time. However, efforts in defect detection were less; the available literatures considered mostly non-destructive techniques, and it suffered from several limitations. Hence, the present work attempts to detect defects in friction-stir-welded samples through real-time temperature signal. The rate of change of temperature and wavelet-analysis-based indicator computed from temperature signal show appreciable contrast between defective and defect-free welds. Effectively, the present approach is free from any post-processing of the welds as required in other detection techniques such as non-destructive methods; it eventually reduces post-operation time and can be effective in enhancing productivity in an actual industrial environment.

\section{Materials and methods}

Single-pass welding procedure on AA 1050 aluminium alloy is used for obtaining the welds in butt joint configuration. The effect of different tool pin profiles is investigated experimentally, keeping the rotational speed and welding speed fixed at a single level. The tool used for the welding experiments is made of SS316 with shoulder diameter fixed at $20 \mathrm{~mm}$. The root diameter of the pin is $6 \mathrm{~mm}$ and length of the pin is $5.7 \mathrm{~mm}$. Tools made for the experiments consist of two different thread profiles. One is the square thread, and the other is the taper thread. The complete design matrix with the weld macrographs is shown in table 1 . The temperature signal during each welding experiment is acquired using K-type thermocouples and dedicated data acquisition hardware at a sampling frequency of $10 \mathrm{~Hz}$. Totally four thermocouples are fixed on advancing and retreating side of the welds up to a depth of $2 \mathrm{~mm}$ along the thickness of the plates. The arrangement of thermocouples on welding plates is schematically shown in figure 1. TC1 and TC2 are the thermocouples attached on the advancing side of the weld and TC 3 and TC4 are the thermocouples attached on the retreating side of the weld at a distance of 1 and $4 \mathrm{~mm}$, respectively, from the shoulder line.

The selection of thermocouples is made keeping the fact in consideration that the initial and final portions of welding process are transient. This transient nature of the process will result in information related to process of formation of defects within the weld. Two zones are identified based on experience for the thermocouples as shown in figure 1. The distance of the thermocouples from the shoulder line is selected to observe the dissipation of heat in the lateral direction to the weld. Heat conduction within the material is a transient process and this can be detected over time at specific locations. Hence, the separating distance between the thermocouple points is kept large $(100 \mathrm{~mm}$, see figure 1) so that the behaviour during heating as well as cooling during the process can be captured. Moreover, during the traversing of the tool, if the thermocouples are kept close to each other, individual effect cannot be captured effectively over distance and time as cross-effect between the thermocouples will come into existence. To avoid this situation, thermocouples are kept at large distance along the welding direction.

In the FSW process, local rate of change of temperature greatly influences the mechanical properties of the welded parts [25]. Microstructure of the welds, which includes grain size, grain boundary character, and coarsening and dissolution of precipitates, is influenced by the temperature distribution within and around the stirred zone in FSW process. Hence, it is important to obtain temperature information during the FSW process. Apart from the mechanical properties of the welds, temperature distribution is also influenced by the defect formation in FSW 
Table 1. Design matrix with pin profiles and weld macrographs.

\begin{tabular}{|c|c|c|c|c|}
\hline $\begin{array}{l}\text { Exp. } \\
\text { no. }\end{array}$ & $\begin{array}{l}\text { Rotational speed } \\
\text { (rev/min) }\end{array}$ & $\begin{array}{l}\text { Welding speed } \\
(\mathrm{mm} / \mathrm{min})\end{array}$ & Pin profile & Weld macrographs \\
\hline E1 & 1100 & 98 & Taper tool & \\
\hline E2 & 1100 & 98 & $\begin{array}{c}\text { Cylindrical tool with } \\
\text { taper thread }\end{array}$ & \\
\hline & & & & \\
\hline E3 & 1100 & 98 & $\begin{array}{c}\text { Taper tool with square } \\
\text { thread }\end{array}$ & \\
\hline E4 & 1100 & 98 & Cylindrical & \\
\hline E5 & 1100 & 98 & $\begin{array}{c}\text { Cylindrical tool with } \\
\text { square thread }\end{array}$ & \\
\hline
\end{tabular}

process and vice-versa. One of the root causes for formation of defects inside the welded samples is inadequate material flow due to poor material plasticization. Along with the deformation of the material around the rotating tool in FSW process, temperature inside the weld pool is also responsible for inadequate plasticization of material [26-29]. Hence, monitoring the real-time temperature signal in FSW process can lead to alternate perspective for defect identification. Moreover, temperature distribution in the welds is the cumulative outcome of the combination of different input process parameters. Individual monitoring of those parameters may not be cost effective. Thus, analysis of temperature signals in real time can open new avenues of defect identification in FSW process.

Time-temperature profiles for experimental cases E1 and E3 are shown in figure 2. It is to be noted that these two experiments are carried out at the same level of process parameters but the weld without threaded pin profile results in internal defect. The temperature signals do not provide any insight that could be an effective indication to reveal the presence of defects inside the weld. In both cases, maximum temperature is obtained on the advancing side of the weld, which is followed by retreating side (from thermocouples at a distance of $1 \mathrm{~mm}$ from the shoulder line). Another observation from the time-temperature profile of the welds indicates that the slope of the curves is high in heating than in cooling. This indicates that rate of change of temperature during heating is much larger than that of cooling phase. This finding can be attributed to the fact that the distance of the thermocouples is kept very close to that of the shoulder line. During welding, the points of the thermocouples are exposed to rapid change of temperature gradient, which is more in case of heating due to the presence of the moving heat source. However, absence of heat source during cooling makes the temperature dissipation process stable and the slope of the cooling curves is relatively low compared with that of heating curves. 


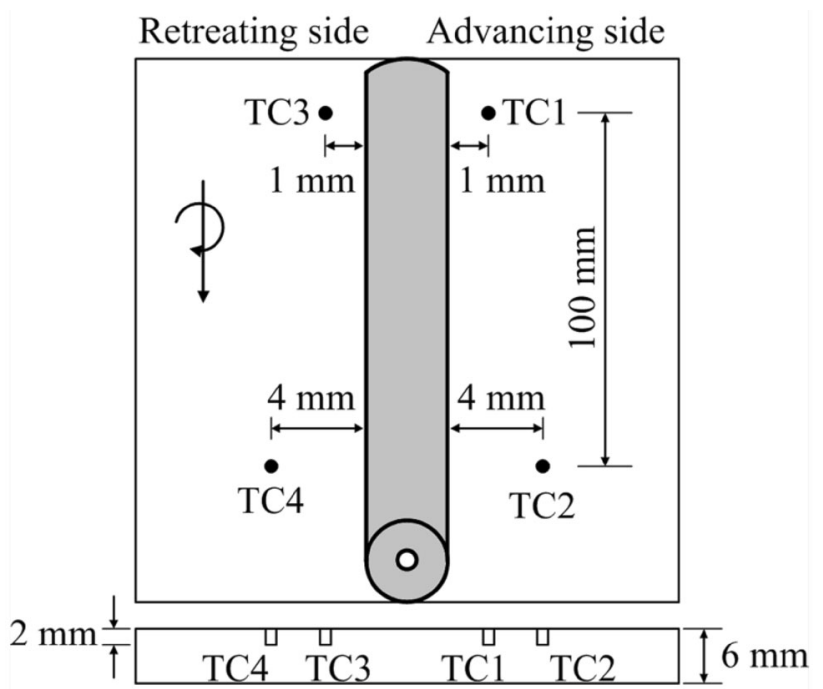

Figure 1. Arrangement of thermocouples in the welding plates.

Inadequacy of fetching valuable information for detection of defects in the welds from time-temperature data motivates this study to investigate rate of change of temperature within the weld. Rate of change of temperature can be treated as a window to observe time-temperature behaviour of the welds, which will capture both heating and cooling information during the welding process. Mathematically, rate of change of temperature $\left(R_{T}\right)$ can be represented as

$$
R_{T}=\left|\frac{T_{i+1}-T_{i}}{t_{i+1}-t_{i}}\right|
$$

where $T$ represents temperature, $t$ represents time and $i$ represents unit step or interval. The mathematical expression of Eq. (1) actually represents the heating rate or cooling rate over fixed space in solution domain. The rate of temperature change links thermal diffusivity, which is the ratio of the time derivative of temperature to its curvature, and essentially it is a measure of thermal inertia. The presence of any discontinuous volume, i.e., internal defects, obviously alters thermal diffusivity and hence can be detected through rate of temperature change.

The present work presents another approach based on DW analysis for the detection of defects using real-time temperature signal. Wavelet analysis is one of the most widely used signal processing techniques. Hence, for a comparative analysis with the proposed approach based on rate of change of temperature, DW analysis is added in this work. Moreover, wavelet analysis is well known for transient detection in signals [30]. As defect formation can be correlated to temperature in the weld, any anomaly is expected to be detected using DW analysis from temperature signal. Temperature signal against each thermocouple and against each experiment is analysed using DW at fourth level and using Daubechies fifth order (db5) mother wavelet function. Theoretical illustration of wavelet analysis is available in literature and can be found in relevant technical articles $[17,31]$. The proposed analysis revolves around computing the detail coefficients of the signals. The detail coefficients are chosen for further analysis as they retain he high-frequency components of the signal and defect formation causes an increase in frequency of the signal [2]. The detail coefficients are further processed and a new indicator is proposed as given in Eq. (2):

$$
I_{W}=\sum_{k=1}^{4}\left[r m s\left(c D_{k}\right)\right]
$$
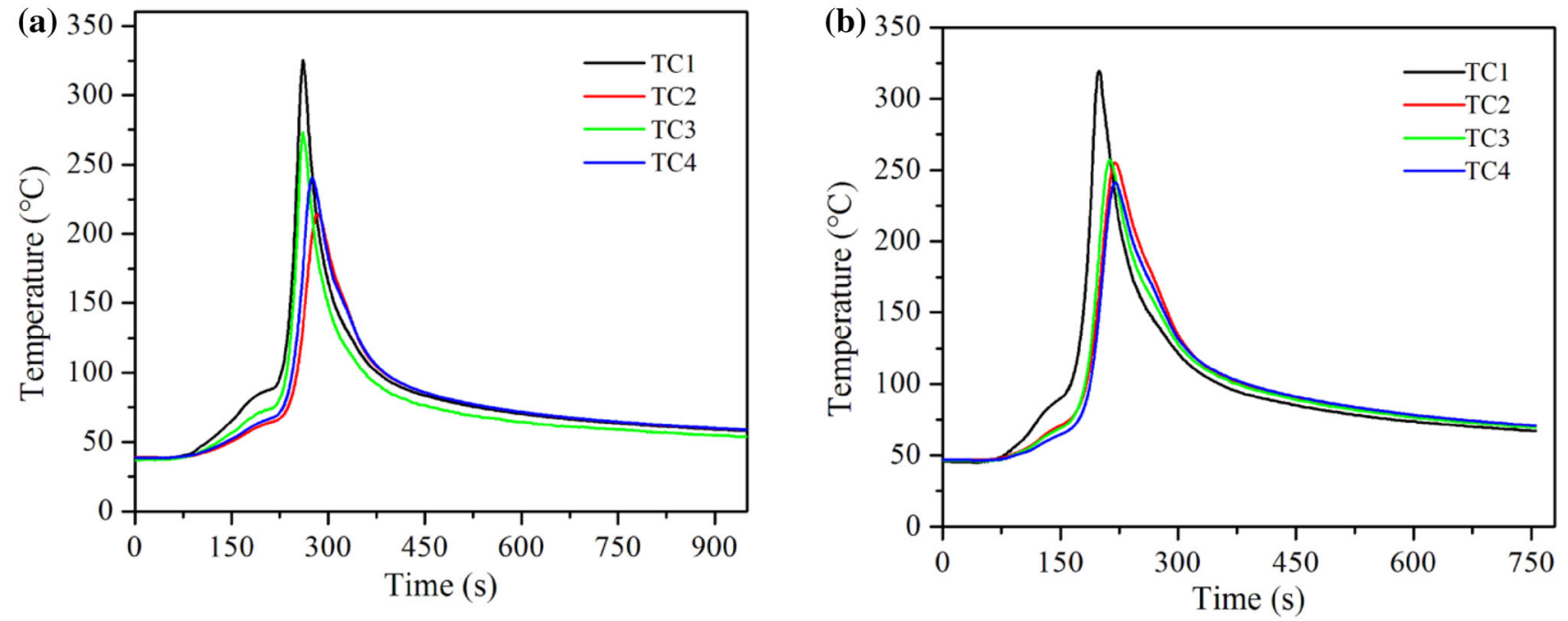

Figure 2. Time-temperature profile of welds from experiments: (a) E1 and (b) E3. 
where $I_{W}$ is the wavelet-based indicator, $c D_{k}$ are the detail coefficients at level $k, i$ is the total number of detail coefficients at level $k$ and $r m s$ stands for the root mean square values of the detail coefficients.

\section{Results and discussion}

Using five experimental conditions (table 1), the analysis is performed. In figure 3 , absolute rate of change of temperature computed through Eq. (1) is displayed against each thermocouple point and for each experimental case. Remarkably, it can be seen from figure 3 that $R_{T}$ of defective weld samples is lower than that of defect-free weld samples. The proposed method leads to an effective notion that the indicator proposed can be an efficient


alternative for detection of defects in friction-stir-welded samples.

The justification for the low $R_{T}$ values for defective welds can be derived from the physics involved in defect formation in FSW process. The defects reported in table 1 (Exp. Nos. E1 and E4) are formed due to insufficient material flow around the pin. Insufficient material flow is the result of inadequate heat generation during the welding process or vice-versa since response (i.e., temperature measurement) is also affected by discontinuity of material volume. The time-temperature information also shows nominal change in maximum temperature for defective and defect-free regions. The temperature change indicates the temperature decay in the welds. Lower $R_{T}$ signifies that rate of decay is lower and vice-a-versa. This slower decay is attributed to the fact that the defect region is devoid of continuity. The void formed is possibly filled
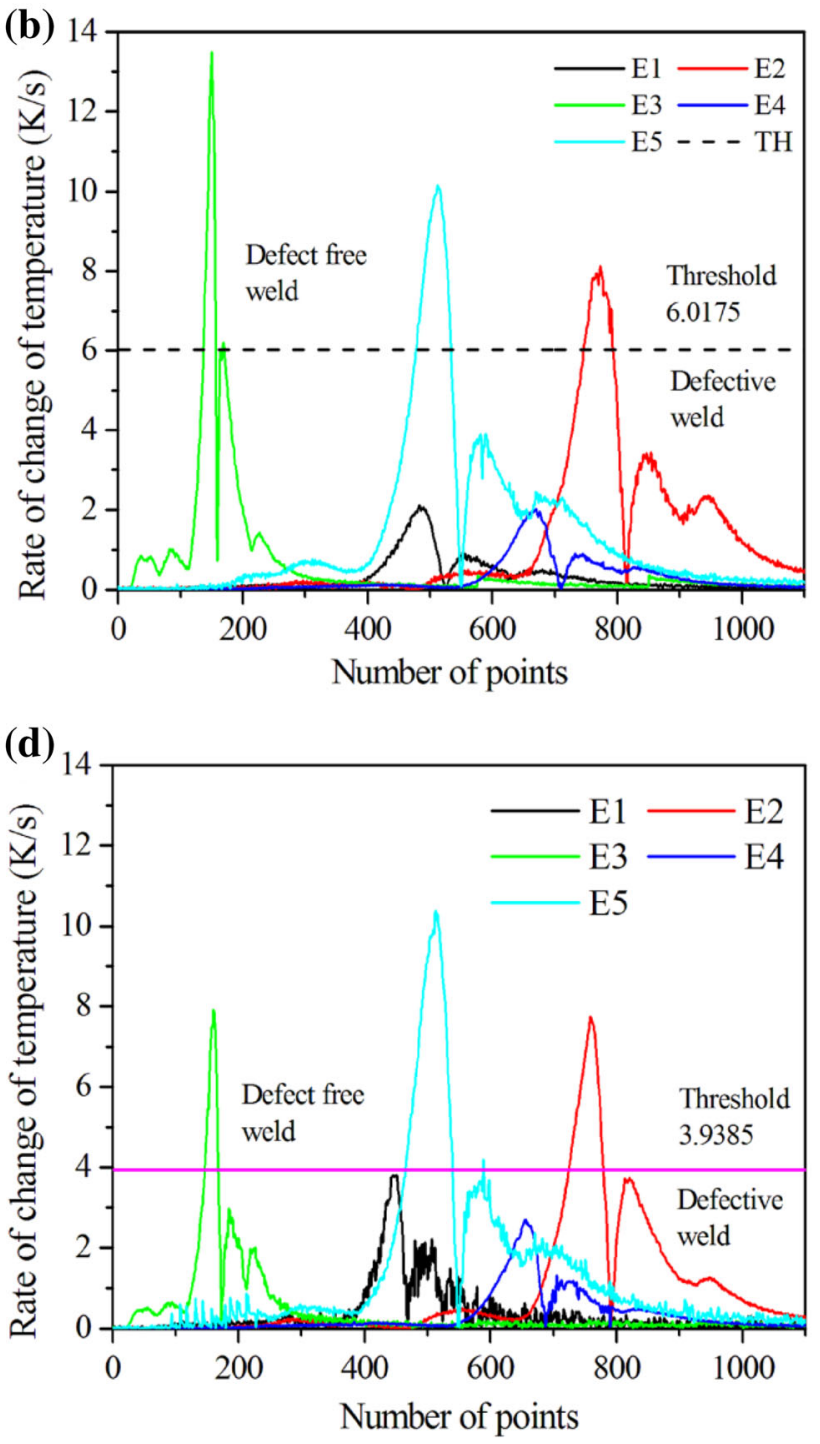

Figure 3. Rate of change of temperature against thermocouples: (a) TC1, (b) TC2, (c) TC3 and (d) TC4. 
with air as contamination of other gaseous elements in FSW process is negligible [32]. Thermal conductivity of air $(\sim 0.0257 \mathrm{~W} / \mathrm{m} \mathrm{K}$ at $293 \mathrm{~K})$ is several times lower than that of aluminium ( $\sim 205 \mathrm{~W} / \mathrm{m} \mathrm{K}$ at $293 \mathrm{~K})$. Hence, temperature of defect region decreases at much slower rate than that of defect-free region. Slow temperature decay in defective weld is also reported by [3]. This effectively justified the lower $R_{T}$ for defective welds than defect-free welds. Thus, the present approach with realtime temperature data rate of change of temperature can be an effective alternative to existing defect detection methodologies.

Observation made from figure 3 is the non-linear behaviour of rate of change of temperature. This can be attributed to the non-linear behaviour of the temperature profiles obtained from the thermocouple data during welding. Owing to thermal properties and heat transfer phenomena in and around the weld zone, the process of heating in the presence of a moving heat source is non-linear. The non-linear behaviour can be observed from $3 \mathrm{D}$ heat conduction equation, which is represented as

$$
\frac{\partial T}{\partial t}-\alpha \nabla^{2} T=0
$$

where $T(x, y, z, t)$ represents temperature, $\alpha$ represents thermal diffusivity of the material and $\nabla^{2}$ the Laplace operator. The relation indicates a non-linear behaviour of the heat transfer phenomena over a certain volume of material. The same can be observed with temperature profiles represented in figure 1. As the temperature perceives a non-linear behaviour; the rate of change of temperature over time (or space) can be observed as nonlinear. This non-linear behaviour of the rate of change of temperature indicates that the heat distribution (heat addition and heat removal) in the weld in the presence as well as absence of moving heat source is also non-linear. This behaviour of the rate of change of temperature is a characteristic of welds with and without internal defects. However, the non-linearity in case of defective welding cases is observed to be comparatively less compared with that of defect-free welding cases. One of the possible reasons for the same is the fact that defect results in material discontinuity within the weld and this further affects the thermal properties of the weld. This is attributed to low rate of change of temperature for the defective welding compared with defect-free welding cases as shown in figure 3.

The computed absolute $R_{T}$ values are further processed to compute the threshold limit for finding the boundary to classify defective welds from defect-free welds. This is achieved by finding the Euclidean distance between the $R_{T}$ points using min-max relationship as shown in Eq. (4). From the analysis, respective threshold limits are computed as shown in figure 3 . These threshold values efficiently differentiate the defective region from defect-free region. The threshold limit is expressed as

$$
\delta_{T}=\operatorname{dist}\left[\min \left\{\max \left(R_{T}^{D F}\right)\right\}, \max \left\{\max \left(R_{T}^{D}\right)\right\}\right]
$$

where 'dist' represents Euclidean distance, $R_{T}^{D F}$ represents temperature gradient for defect-free welding and $R_{T}^{D}$ represents temperature gradient against defective welding. From this equation, $\delta_{L}$ values against thermocouples TC1, TC2, TC3 and TC4 are found to be $12.45,6.12,7.23$ and 5.05 , respectively.

Wavelet decomposition of the temperature signal (against thermocouple TC1, see figure 1) acquired during Exp. Nos. E4 and E5 are shown in figure 4. It is evidential that detail coefficients have a different pattern for defective welding than defect-free welding case. This is further reflected in the proposed indicator computed using Eq. (2). The values of the proposed indicator are shown in figure 5 . The figure displaying the $I_{W}$ reveals a clear boundary for classifying defective welds from defect-free welds. The difference that persits in detail coefficients for defective welds and defect-free welds is well captured through computing the proposed wavelet-based indicators. It is observed that the proposed indicators for defect-free welding cases are quite low compared with defective welding cases. This can be justified with the physical illustration of DW analysis.

In DW analysis, the original signal is decomposed into approximation and detail parts relating to the sampling frequency of the signals, where approximation part contains the low-frequency components of the signal and detail part contains the high-frequency components. From the literature it is observed that inclusion of defects in FSW process changes signal frequency to higher scales. Moreover, the amplitude of the detail coefficient for defective welding cases is also high as compared with defect-free welding cases. This reflects that high-frequency components of the signals against defective cases have high spatial distribution than signals against the defect-free welding. The proposed wavelet-based indicators are efficient in capturing these change in amplitude values and reflect an efficacious representation of defect conditions within the welds. This non-destructive evaluation approach is simple and free from post-processing steps involved in other available non-destructive evaluation techniques. The computations of $I_{W}$ can be integrated to hardware for less human intervention, making the proposed approach to be less dependent on operator skill.

The proposed $I_{W}$ can be effectively used for finding threshold values for classifying defective and defect-free welds. In this work, threshold value for differentiating the defective weld zone from defect-free weld zone is computed from Euclidean distance calculation in the waveletbased indicators. The method of threshold computation is expressed as 
(a)
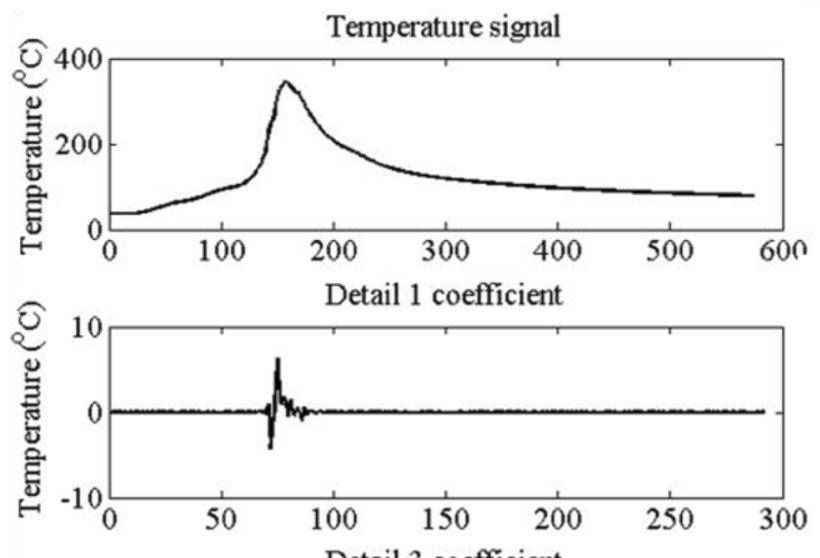

Detail 3 coefficient

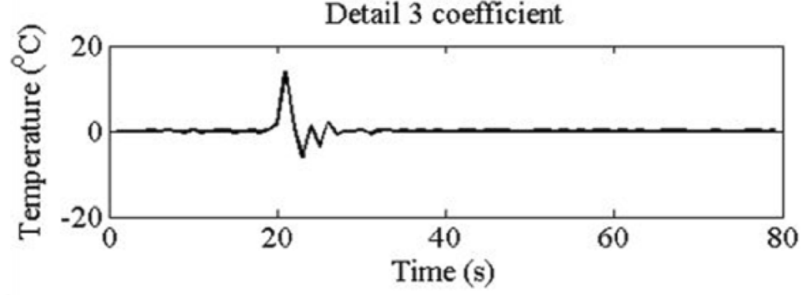

(b)
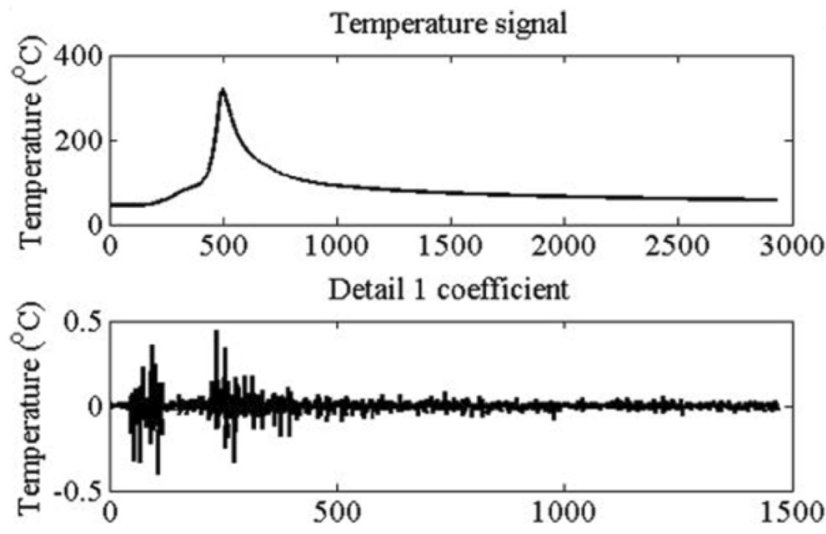

Detail 3 coefficient

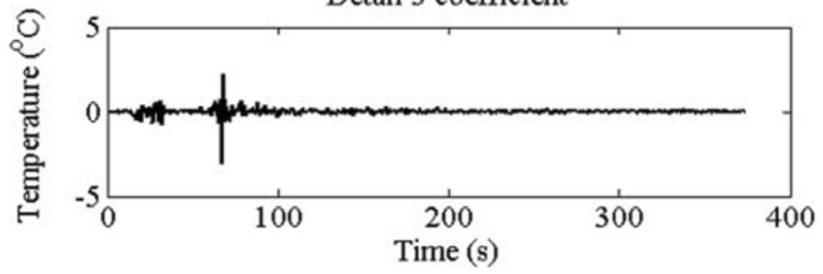

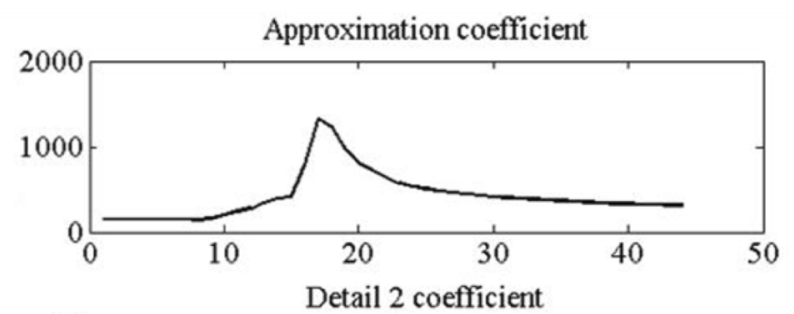
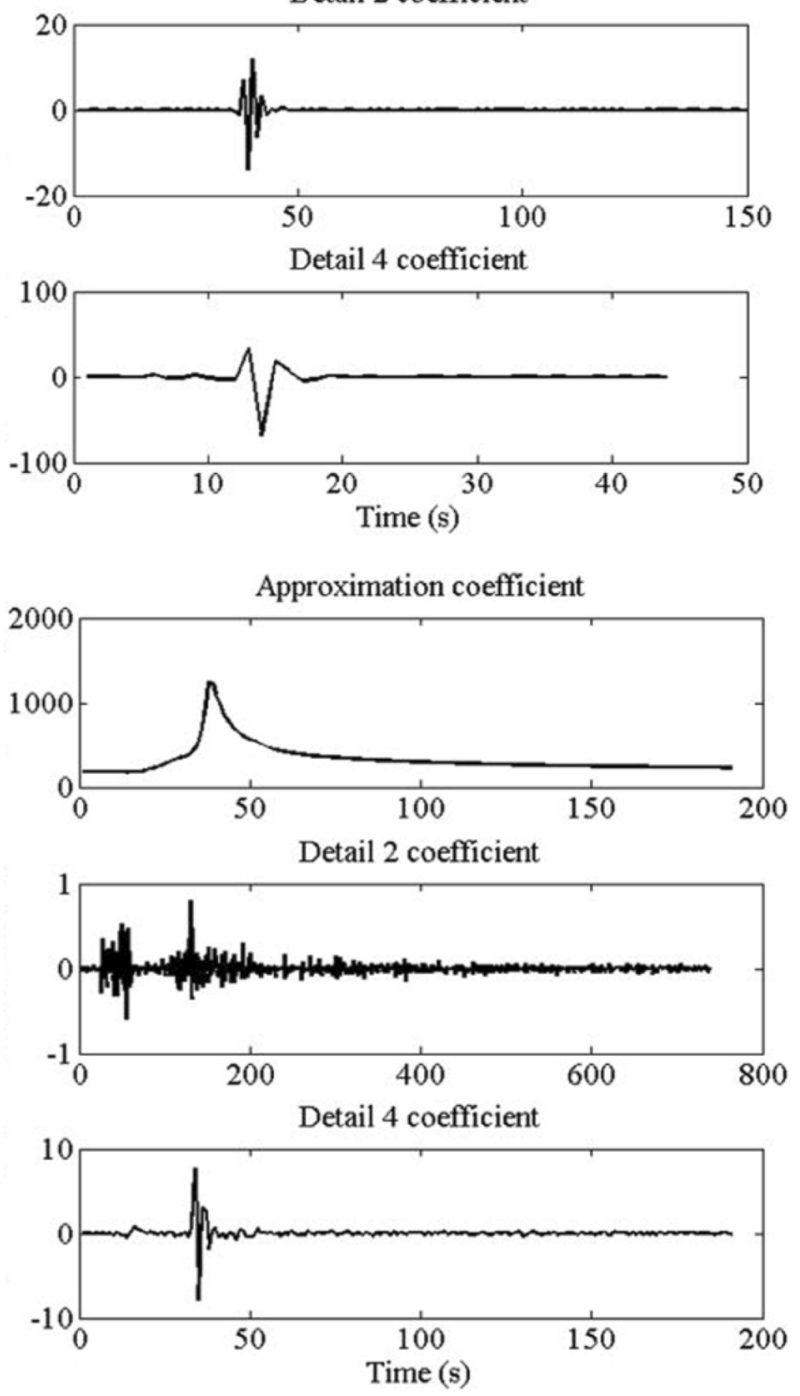

Figure 4. Wavelet decomposition of temperature signal for TC1 against (a) Exp. No. 4 and (b) Exp. No. 5.

$$
\left.\delta_{W}=\operatorname{dist}\left[\max \left(I_{W}^{D F}\right), \min \left(I_{W}^{D}\right)\right)\right]
$$

where $I_{W}^{D F}$ represents wavelet-based indicators for defectfree welding cluster and $I_{W}^{D}$ for the defective welding cluster. For the present case, $\delta_{W}$ is found out to be 4.58 . This threshold limit sets a clear boundary for the classification of defective welds from defect-free welds as shown in figure 5.
The discussion and results furnished in this research work are based on the limited range of process parameters and tool geometries. A wide range of process parameters with different tool geometries needs to be considered for the generalization of the results applicable to FSW process, which is the future scope of this research work. The proposed approach can be the first level of safeguard regarding the detection of presence of defects in FSW process. 


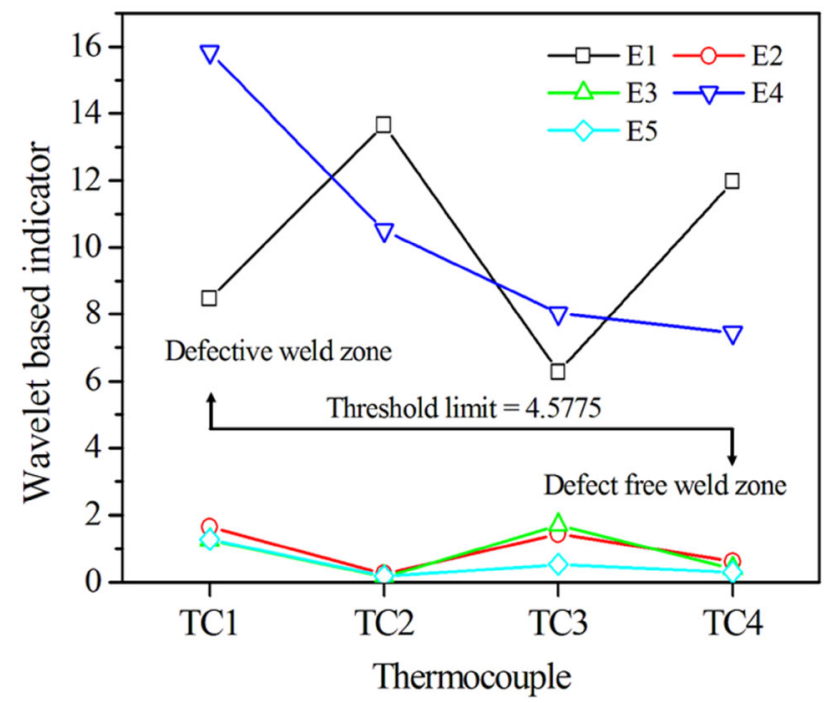

Figure 5. Wavelet-based indicator against experiments.

\section{Conclusions}

Effect of different pin profiles on defect formation in FSW process is investigated experimentally. From the investigation, it is observed that tool pin without threads yields defective welds. Real-time temperature signals during the experiments are acquired using K-type thermocouples and dedicated data acquisition hardware. Two approaches for detection of subsurface defect in FSW process are presented using temperature signals. Rate of change of temperature and wavelet-based indicator are the two new indicators proposed for effective detection of defects in FSW process. From the analysis it is found that rate of temperature change for defective welds is much lower than the ones for defect-free welds. Wavelet-based indicators for defective welds are higher than those for defect-free welding cases. Threshold values for rate of change of temperature and wavelet-based indicators are computed through Euclidean distance for the respective cases. The computed threshold values effectively set a boundary for the differentiation of defective welds from the defect-free welds. These salient findings can be effective for monitoring of FSW process through setting feasible threshold values for detection of defect formation during the process. The approach presented requires less human intervention, making it suitable for intelligent systems for decision making regarding the outcome of the process.

\section{Acknowledgements}

The authors gratefully acknowledge the financial support provided by SERB (Science and Engineering Research Board), India (Grant No. SERB/F/2767/2012-13), to carry out this research work.

\section{References}

[1] Kim Y G, Fujii H, Tsumura T, et al 2006 Three defect types in friction stir welding of aluminium die casting alloy. Mater. Sci. Eng. A 415: 250-254

[2] Boldsaikhan E, Corwin E M, Logar A M and Arbegast W J 2011 The use of neural network and discrete Fourier transform for real-time evaluation of friction stir welding. Appl. Soft Comput. 11: 4839-4846

[3] Saravanan T, Lahiri B B, Arunmuthu K, et al 2014 Nondestructive evaluation of friction stir welded joints by X-ray radiography and infrared thermography. Proc. Eng. 86: 469-475

[4] Chen H B, Yan K and Lin T 2006 The investigation of typical welding defects for 5456 aluminium alloy friction stir welds. Mater. Sci. Eng. A 433: 64-69

[5] Rosado L S, Santos T G, Piedade M, et al 2010 Advanced technique for non-destructive testing of friction stir welding of metals. Measurement 43: 1021-1030

[6] Iwaki S, Okada T, Eguchi N, Tanaka S, Namba K and Oiwa N 2006 Imperfections in friction stir welded zones and their precision non-destructive testing. Studies on characteristics of friction stir welded joints in structural thin aluminium alloys. Weld. Int. 20: 197-205

[7] Cosmi F, Cristofori A and Mancini L 2005 Preliminary investigation by synchrotron radiation of cracks and defects in AA FSW samples. In: Proceedings of the 11th International Conference on Fracture, Paper No. 5450, Turin, Italy

[8] Moles M, Lamarre A and Cancre F 2002 Utilization of stateof-the-art phased array inspection technology for the evaluation of friction stir welds. In: Proceedings of NDE 2002: Predict, Assure, Improve, Chennai, India

[9] Moles M, Lamarre A and Dupuis O 2004 Complete inspection of friction stir welds in aluminium using ultrasonic and eddy currents arrays. In: Proceedings of 16th WCNDT 2004 World Conference on NDT, Montreal Canada, Paper No 84

[10] Wu C S, Wang L, Ren W J and Zhang X Y 2014 Plasma arc welding: process, sensing, control and modeling. J. Manuf. Process. 16(1): 74-85

[11] Huang X and Chen S 2006 SVM-based fuzzy modeling for the arc welding process. Mater. Sci. Eng. A 427(1-2): 181-187

[12] He K, Li Q and Chen J 2013 An arc stability evaluation approach for SW AC SAW based on Lyapunov exponent of welding current. Measurement 46(1): 272-278

[13] Zhang Z, Chen H, Xu Y, Zhong J, Lv N and Chen S 2015 Multisensor-based real-time quality monitoring by means of feature extraction, selection and modelling for Al alloy in arc welding. Mech. Syst. Signal Process. 60-61: 151-165

[14] Cavaliere P, Campanile G, Panella F, et al 2006 Effect of welding parameters on mechanical and microstructural properties of AA6056 joints produced by friction stir welding. J. Mater. Process. Technol. 180: 263-270

[15] Arora K S, Pandey S, Schaper M and Kumar R 2010 Effect of process parameters on friction stir welding of aluminum alloy 2219-T87. Int. J. Adv. Manuf. Technol. 50: 941-952

[16] Yang Y, Kalya P and Landers R G 2008 Automatic gap detection in friction stir butt welding operations. Int. J. Mach. Tool. Manuf. 48: 1161-1169 
[17] Chen C, Kovacevic R and Jandgric D 2003 Wavelet transform analysis of acoustic emission in monitoring friction stir welding of 6061 aluminium. Int. J. Mach. Tool. Manuf. 43: $1383-1390$

[18] Soundararajan V, Atharifar H and Kovacevic R 2006 Monitoring and processing the acoustic emission signals from the friction stir welding process. Proc. IMechE Part B J. Eng. Manuf. 220: 1673-1685

[19] Mehta M, Chatterjee K and De A 2013 Monitoring torque and traverse force in friction stir welding from input electrical signatures of driving motors. Sci. Technol. Weld. Join. 18: $191-197$

[20] Das B, Pal S and Bag S 2014 Monitoring of friction stir welding process through signals acquired during the welding. In: Proceedings of the 5th International and 26th All India Manufacturing, Design and Research Conference (AIMTDR2014), December 12-14, 2014, Assam, India

[21] Bhat N N, Kumari K and Dutta S 2015 Friction stir weld classification by applying wavelet analysis and support vector machine on weld surface images. J. Manuf. Process. 20(1): 274-281

[22] Das B, Pal S and Bag S 2016 Defect detection in friction stir welding process through characterization of signals by fractal dimension. Manuf. Lett. 7: 6-10

[23] Shrivastava A, Dingler C, Zinn M and Pfefferkorn F E 2015 Physics-based interpretation of tool-workpiece interface temperature signals for detection of defect formation during friction stir welding. Manuf. Lett. 5: 7-11

[24] Khandkar M Z H, Khan J A and Reynolds A P 2003 Prediction of temperature distribution and thermal history during friction stir welding: input torque based model. Sci. Technol. Weld. Join. 8(3): 165-174

[25] Fuller M D, Swaminathan S, Zhilyaev A P and McNelley T R 2007 Microstructural transformations and mechanical properties of cast NiAl bronze: effects of fusion welding and friction stir processing. Mater. Sci. Eng. A 463: 128-137

[26] Shirazi H, Kheirandish S and Safarkhanian M A 2015 Effect of process parameters on the macrostructure and defect formation in friction stir lap welding of AA5456 aluminium alloy. Measurement 76: 62-69

[27] Chen Z W, Pasang T and Qi Y 2008 Shear flow and formation of nugget zone during friction stir welding of aluminium alloy 5083-O. Mater. Sci. Eng. A 474: 312-316

[28] Saeid T, Abdollah-zadeh A, Assadi H, et al 2008 Effect of friction stir welding speed on microstructure and mechanical properties of a duplex stainless steel. Mater. Sci. Eng. A 496: 262-268

[29] Morisada Y, Imaizumi T and Fujii H 2015 Clarification of material flow and defect formation during friction stir welding. Sci. Technol. Weld. Join. 20: 130-137

[30] Guasp M R, Daviu A A, Sanchez M P, Panadero R P and Cruz J P 2008 A general approach for transient detection of slip-dependent fault components based on the discrete wavelet transform. IEEE Trans. Ind. Electron. 55: 4167-4180

[31] Pal S, Pal S K and Samantaray A K 2008 Neurowavelet packet analysis based on current signature for weld joint strength prediction in pulsed metal inert gas welding process. Sci. Technol. Weld. Join. 13: 638-645

[32] Mishra R S and Ma Z Y 2005 Friction stir welding and processing. Mater. Sci. Eng. R 50: 1-78 